\title{
A Novel Screening Strategy Reveals ROS-Generating Antimicrobials That Act Synergistically against the Intracellular Veterinary Pathogen Rhodococcus equi
}

\author{
Álvaro Mourenza ${ }^{\circledR}$, José A. Gil ${ }^{\mathbb{D}}$, Luís M. Mateos * and Michal Letek *D \\ Department of Molecular Biology, Area of Microbiology, University of León, 24071 León, Spain; \\ amouf@unileon.es (Á.M.); jagils@unileon.es (J.A.G.) \\ * Correspondence: luis.mateos@unileon.es (L.M.M.); michal.letek@unileon.es (M.L.)
}

Received: 31 December 2019; Accepted: 26 January 2020; Published: 28 January 2020

\begin{abstract}
Rhodococcus equi is a facultative intracellular pathogen that causes infections in foals and many other animals such as pigs, cattle, sheep, and goats. Antibiotic resistance is rapidly rising in horse farms, which makes ineffective current antibiotic treatments based on a combination of macrolides and rifampicin. Therefore, new therapeutic strategies are urgently needed to treat $R$. equi infections caused by antimicrobial resistant strains. Here, we employed a R. equi mycoredoxin-null mutant strain highly susceptible to oxidative stress to screen for novel ROS-generating antibiotics. Then, we used the well-characterized Mrx1-roGFP2 biosensor to confirm the redox stress generated by the most promising antimicrobial agents identified in our screening. Our results suggest that different combinations of antibacterial compounds that elicit oxidative stress are promising anti-infective strategies against R. equi. In particular, the combination of macrolides with ROS-generating antimicrobial compounds such as norfloxacin act synergistically to produce a potent antibacterial effect against $R$. equi. Therefore, our screening approach could be applied to identify novel ROS-inspired therapeutic strategies against intracellular pathogens.
\end{abstract}

Keywords: Rhodococcus equi; antimicrobials; oxidative stress; macrophages; roGFP2

\section{Introduction}

Rhodococcus equi is an intracellular pathogen that causes granulomatous infections in a wide range of animal species, including foals, dogs, pigs, cattle, sheep, and goats [1-3]. In addition, $R$. equi can infect immunocompromised humans causing a fatal pyogranulomatous bronchopneumonia [4]. R. equi is a widely distributed pathogen, and is frequently endemic in horse farms $[5,6]$. This pathogen is usually transmitted by inhaling aerosol respiratory particles or dust contaminated with $R$. equi $[5,6]$.

During the last decade, the number of $R$. equi isolates resistant to commonly used antimicrobials has dramatically increased [7-9], making current antibiotherapies often ineffective [10]. Primary preventative strategies based on the use of vaccines or hyperimmune plasma administration do not confer full protection against this pathogen [11-13]. Thus, new treatments based on a combination of clinically established antimicrobials with novel anti-R. equi compounds could be the only realistic option to solve in the short term the crisis caused by antimicrobial resistant strains.

Interestingly, some antimicrobial agents can stimulate the production of reactive oxygen species (ROS) as part of their mechanism of action [14-16]. Therefore, the antioxidant systems of bacterial pathogens could be important to counteract antibiotic ROS production. We recently demonstrated that mycoredoxins are key factors for the maintenance of $R$. equi's redox homeostasis [17]. In particular, a R. equi mutant strain lacking three mycoredoxin-encoding genes (R. equi $\Delta m r x 1 \Delta m r x 2 \Delta m r x 3)$ shows high susceptibility to oxidative stressing agents, including hydrogen peroxide, sodium hypochlorite, 
or nitric oxide [17]. Based on this evidence, we used here this triple $m r x$-null mutant strain of $R$. equi to screen for novel ROS-generating antimicrobial compounds. As a result, we identified potent and synergistic antimicrobial activities of different ROS-producing antibiotics against $R$. equi. In addition, we established a novel screening approach for the identification of ROS-inspired antimicrobial strategies against this intracellular veterinary pathogen.

\section{Materials and Methods}

\subsection{Bacterial Strains, Reagents, and Antibiotic Susceptibility Tests}

Rhodococcus equi $103 S^{+}$was kindly provided by Dr. Jesús Navas from the University of Cantabria (Spain), which was considered the wild type strain in all assays. R. equi $\Delta m r x 1 \Delta m r x 2 \Delta m r x 3$ and $R$. equi $\Delta m r x 1 \Delta m r x 2 \Delta m r x 3$ expressing $m r x 1$-roGFP2 are derivative strains of $R$. equi 103S ${ }^{+}$[17]. All reagents were procured from Sigma-Aldrich, unless otherwise stated.

For antibiotic susceptibility tests, exponential growth phase cultures $\left(\mathrm{OD}_{600}=1\right)$ of $R$. equi were diluted in melted (at $50^{\circ} \mathrm{C}$ ) trypticase soy agar (TSA) plates at 1:10 mL dilution and spread over $10 \mathrm{~mL}$ solid TSA plates. Disks with antibiotics were then placed onto R. equi-containing TSA plates, which were incubated at $30^{\circ} \mathrm{C}$ for $24 \mathrm{~h}$.

\subsection{In vivo Quantification of Redox Homeostasis}

The biosensor Mrx1-roGFP2 [17] was used to evaluate the intracellular Redox potential of R. equi $103 S^{+}$during infection. The redox status of Mrx1-roGFP2 was measured as described previously [17]. Briefly, pellets of exponentially growing $R$. equi $\Delta m r x 1 \Delta m r x 2 \Delta m r x 3$ expressing $m r x 1-r o G F P 2$ [17] were resuspended in Phosphate-Buffered Saline (PBS) with different concentrations of antibiotics, treated with 10\% N-Ethylmaleimide (NEM) to block free thiol groups, and fixed with $70 \%$ ethanol on poly-L-lysine-treated microscope slides. In parallel, the Mrx1-roGFP2 total reduction or oxidation status was calculated by adding $40 \mathrm{mM}$ dithiothreitol (DTT) or $10 \mathrm{mM}$ of diamide (DIA), respectively.

In all cases, 200 bacterial cells from different treatments were evaluated on a Zeiss LSM800 confocal microscope with Airyscan as previously described [17-19]. The ratio between fluorescence emission at $530 \mathrm{~nm}$ after excitation at 405 (oxidized) and $490 \mathrm{~nm}$ (reduced) was calculated pixel by pixel with ImageJ (http://rsb.info.nih.gov/ij/). The quantification of redox potentials was performed as described by Gutscher et al. [20].

\subsection{Minimum Inhibitory Concentration (MIC) Assays}

The MIC of each antibiotic or combination of antibiotics was calculated as previously described [21]. Briefly, exponentially growing R. equi $103 S^{+}$in a Muller-Hinton broth medium $\left(\mathrm{OD}_{600}=1\right)$ was diluted to obtain a final concentration of $2 \times 10^{5}$ cells in $100 \mu$ per well of microtiter plates of 96-wells. Different concentrations of serially-diluted antibiotics were added to the wells in triplicates and the plate was incubated at $37^{\circ} \mathrm{C}$ during $16 \mathrm{~h}$. A negative control with plain Muller-Hinton broth medium was included in all experiments.

\subsection{Macrophage Survival Test in the Presence of Different Antibacterial Compounds}

Host cell infection assays were performed as described previously [22] using low-passage J774.A1 murine macrophages (ATCC) cultured in Dulbecco's Modified Eagle Media (DMEM -Thermo-Fischer Scientific, Waltham, MA, USA). Briefly, macrophages were infected at a multiplicity of infection of $10(\mathrm{MOI}=10)$ with R. equi $103 \mathrm{~S}^{+}$. After $1 \mathrm{~h}$ of incubation, the medium was replaced with DMEM supplemented with $100 \mu \mathrm{g} / \mathrm{mL}$ gentamicin to kill extracellular bacteria. After $1 \mathrm{~h}$ of incubation in DMEM with gentamicin, cells were washed three times with PBS and incubated with DMEM supplemented with specific antibacterial compounds at different concentrations. After $8 \mathrm{~h}$, macrophages were lysed with $0.1 \%$ Triton X-100 and serial dilutions of the lysates were spread onto TSA plates for R. equi's 
colony forming unit (CFU) counting. The presence of the virulence plasmid pVAPA in R. equi was routinely verified by PCR amplification as previously described [17].

\subsection{Statistical Analyses}

Statistical analyses were conducted using IBM ${ }^{\circledR}$ SPSS ${ }^{\circledR}$ statistics v24 (IBM, ArmonK, New York, NY, USA). Firstly, data distribution was assessed with the Kolmogorov-Smirnov test. If data followed a normal distribution, two-way ANOVA and post-hoc Tukey's multiple-comparison tests were employed to identify statistically significant differences across conditions. When the data were not following a normal distribution, a non-parametric analysis was performed employing a Kruskall-Wallis test.

\section{Results and Discussion}

\subsection{Identification of ROS-Generating Antibacterial Compounds Active against R. equi}

R. equi is well equipped with antioxidant molecules and enzymes [22], which might confer a natural resistance to ROS-generating antimicrobials. Low molecular weight thiols are part of one of the most important antioxidant strategies in bacteria. Mycothiol (MSH) is a low molecular weight thiol unique of actinobacteria [23]. Mycothiol protects proteins from irreversible cysteine oxidation during oxidative stress by forming disulfides with protein thiols. Mycoredoxins (Mrxs) are then required for the reduction of S-mycothiolated proteins to restore their function. Mycoredoxins become S-mycothiolated during the process of de-mycothiolation, and mycothiol restores their reduced state by generating mycothione (MSSM) in the process. Mycothione is then reduced back to mycothiol by an NADPH-dependent mycothione reductase (Mtr).

The $m r x$-null mutant strain R. equi $\Delta m r x 1 \Delta m r x 2 \Delta m r x 3$ lacks three genes encoding for mycoredoxins, which made it highly susceptible to oxidative stress [17]. Due to this, we considered $R$. equi $\Delta m r x 1 \Delta m r x 2 \Delta m r x 3$ a very suitable reporter for screenings aimed at identifying ROS-generating antimicrobials. Thus, we compared the susceptibility of R. equi $103 S^{+}$and R. equi $\Delta m r x 1 \Delta m r x 2 \Delta m r x 3$ to different antibacterial compounds.

We first analysed by the Kirby-Bauer disk diffusion method [24] the growth inhibition area generated by different antibiotics (Figure 1A). We did not observe any halos of inhibition when oxacillin, ampicillin, or fosfomycin trometamol were used in our screen, suggesting that the natural resistance of $R$. equi to these compounds was unrelated to mechanisms implicated in redox homeostasis. On the other hand, the susceptibility of R. equi $103 S^{+}$and R. equi $\Delta m r x 1 \Delta m r x 2 \Delta m r x 3$ to chloramphenicol and clindamycin was apparently analogous (Figure 1A), suggesting that these antibiotics did not generate ROS. However, the halo of inhibition generated by rifampicin, erythromycin, and vancomycin was significantly higher in the triple $m r x$-deletion mutant strain when compared to the wild type strain (Figure 1A). Interestingly, the combination of rifampicin and erythromycin is one of the most commonly used combinatorial antibiotherapy to treat R. equi infections [10]. Therefore, our results suggested that ROS synthesis might have an important role in the mechanism of action of this therapeutic strategy.

To confirm that our initial screening results were effectively due to ROS biosynthesis, we employed the well-characterized Mrx1-roGFP2 biosensor [17] to evaluate the oxidative stress generated in $R$. equi by very low doses of rifampicin, erythromycin, and vancomycin (Figure 1B). It has been recently demonstrated that the redox state of Mrx1-roGFP2 is in direct equilibrium with mycothiol/mycothione levels $[17,19]$. Moreover, Mrx1-roGFP2 could be used to evaluate in real-time the intracellular redox status of bacteria expressing the reporter [17].

Importantly, all three antibiotics generated clear oxidation peaks in Mrx1-roGFP2 (Figure 1B). In particular, we observed an oxidation peak starting at $5 \mathrm{~min}$ of exposure to rifampicin and erythromycin, whereas the oxidation peak caused by vancomycin was only detected after $10 \mathrm{~min}$. These results suggested that the mechanism(s) of ROS biosynthesis of vancomycin might be different to those of rifampicin and erythromycin. In addition, the ratiometric responses of Mrx1-roGFP2 to 
these antibiotics validated our screening approach based on the high susceptibility of the $R$. equi $\Delta m r x 1 \Delta m r x 2 \Delta m r x 3$ strain to oxidative stress.

A

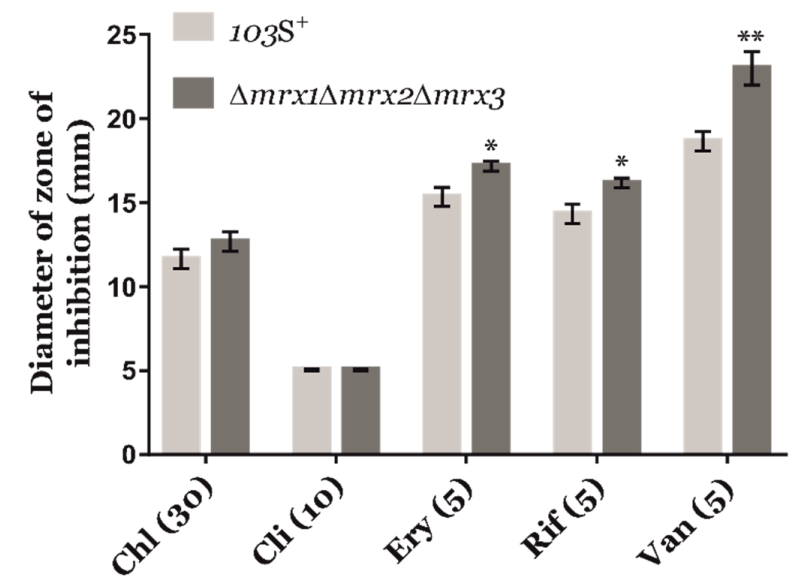

B
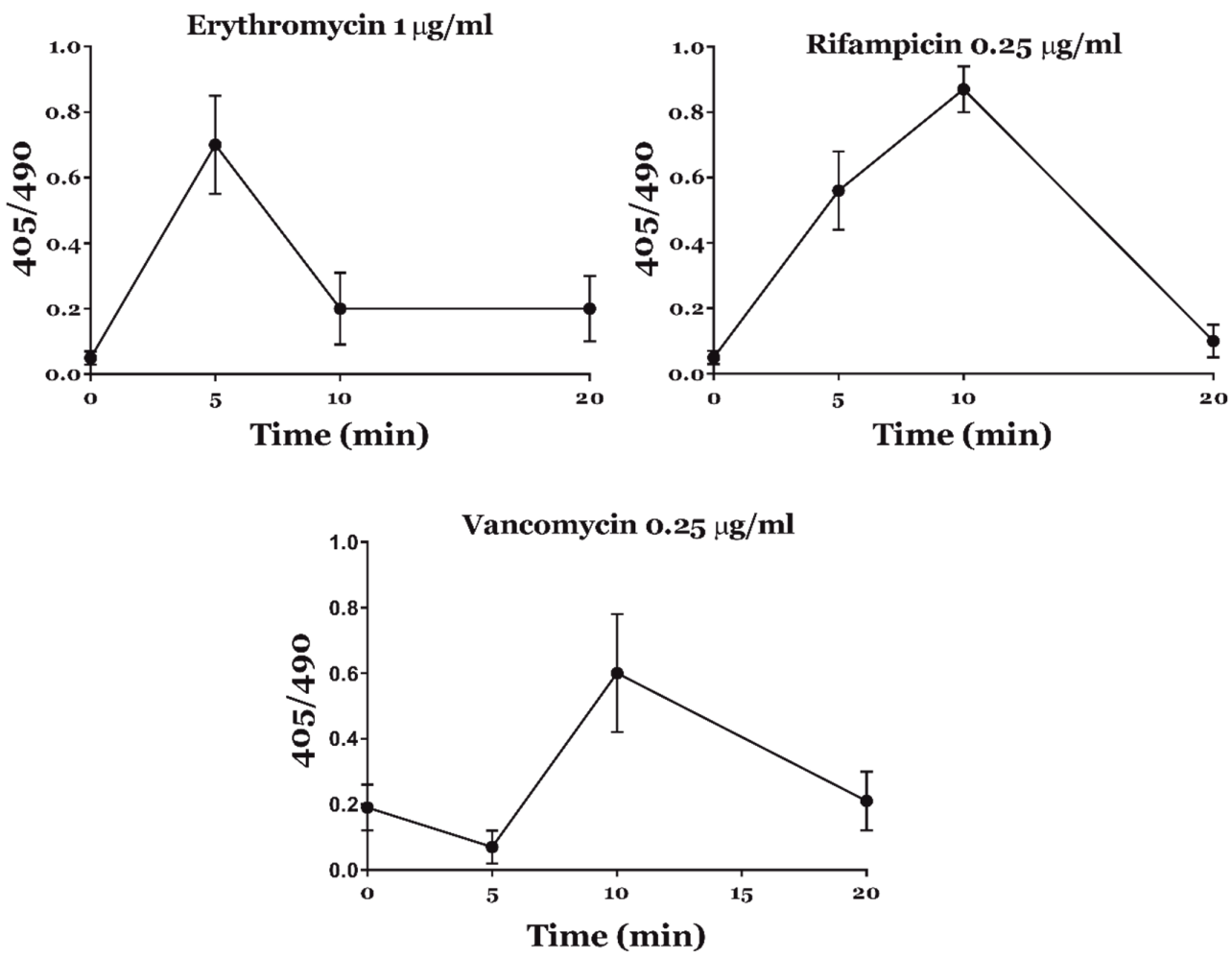

Figure 1. Screening results for the identification of ROS-generating antimicrobials active against $R$. equi. (A) Antimicrobial susceptibility of R. equi $103 S^{+}$(clear grey) and R. equi $\Delta m r x 1 \Delta m r x 2 \Delta m r x 3$ mutant (dark grey) to different antibacterial compounds: Chloramphenicol (Chl), clindamycin (Cli), erythromycin (Ery), rifampicin (Rif), and vancomycin (Van). The concentration used of each antibiotic is shown in brackets $(\mu \mathrm{g} / \mathrm{mL})$. The diameter of the growth inhibition zones was measured to the nearest millimetre, and the mean \pm SD of three independent experiments was plotted. A Kruskall-Wallis non-parametric analysis was performed to test for statistical significance across comparisons of the wild type strain and the triple $m r x$-null mutant. $p$-value $<0.05\left(^{*}\right)$ or $p$-value $<0.01\left(^{* *}\right)$. (B) Ratiometric response of the Mrx1-roGFP2 biosensor. Fluorescence 405/490 ratio was calculated by confocal microscopy at different time points in response to very low doses of rifampicin, erythromycin, and vancomycin. The results show the mean \pm SD of three independent experiments. 
On the other hand, the redox potential ( $E_{\text {roGFP2 }}$ ) of Mrx1-roGFP2 was $-254 \mathrm{mV}$ in response to rifampicin, $-261 \mathrm{mV}$ in erythromycin, and $-273 \mathrm{mV}$ in vancomycin. To put this in context, the basal redox potential of this biosensor was established recently as $-290 \mathrm{mV}$ [17], whereas the $E_{\text {roGFP2 }}$ of Mrx1-roGFP2 in response to $5 \mathrm{mM} \mathrm{H}_{2} \mathrm{O}_{2}$ was $-264 \mathrm{mV}$ and the intracellular redox potential during host cell infection was $-260 \mathrm{mV}$. Overall, these results confirmed that rifampicin, erythromycin, and vancomycin elicited a significant oxidative stress as part of their mechanism of action.

In an attempt to identify other ROS-generating antimicrobials with repurposing potential, we expanded the list of antibiotics tested in our screen with four additional compounds whose ROS-generating mechanism of action has been previously described in other pathogens: Artemisinin, clofazimine, norfloxacin, and nitrofurantoin [14,16,25] (Figure 2). We first compared the effect of these ROS-generating antimicrobials against $R$. equi $103 S^{+}$and $R$. equi $\Delta m r x 1 \Delta m r x 2 \Delta m r x 3$ by means of the disk diffusion method. We did not observe any halos of inhibition with artemisinin and nitrofurantoin, suggesting that the resistance of $R$. equi to these compounds is unrelated to redox stress. Interestingly, R. equi was susceptible to the ROS-generating anti-tuberculosis drug clofazimine [26] (Figure 2A). However, we did not observe any difference in the susceptibility of $R$. equi $\Delta m r \times 1 \Delta m r \times 2 \Delta m r x 3$ to this compound when compared to the wild type strain, which suggested that the mechanism of action of clofazimine in $R$. equi was not mediated by redox stress. Importantly, the zone of inhibition of norfloxacin was significantly higher for $R$. equi $\Delta m r x 1 \Delta m r x 2 \Delta m r x 3$ when compared to $R$. equi $103 S^{+}$(Figure 2A). Moreover, we observed a clear peak of oxidation in Mrx1-roGFP2 after only $3 \mathrm{~min}$ of exposure to norfloxacin (Figure 2B), and the redox potential of Mrx1-roGFP2 was $-278 \mathrm{mV}$. Taken together, these results suggested that one of the mechanisms of action of norfloxacin was mediated by redox stress in $R$. equi, most likely by the generation of oxidized nucleotides within the bacterial cell $[25,27,28]$.

A

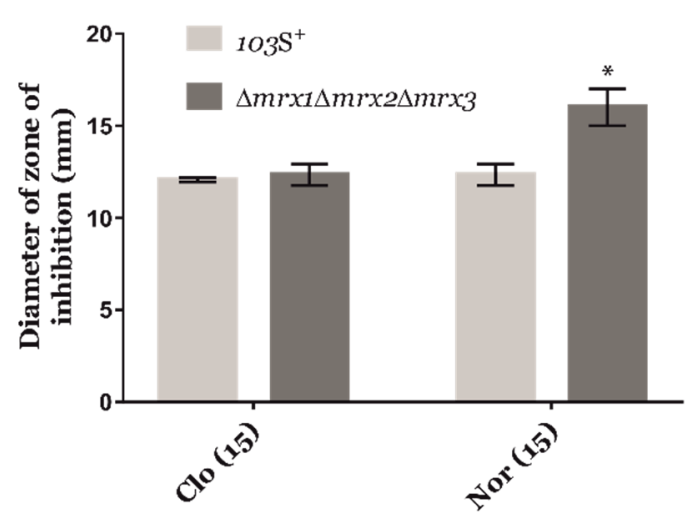

B

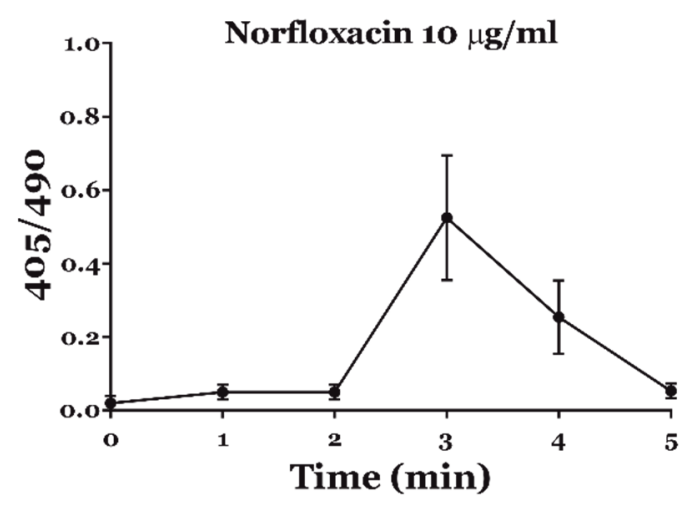

Figure 2. Identification of other ROS-generating antimicrobials against R. equi. (A) Antimicrobial susceptibility of R. equi $103 S^{+}$(clear grey) and R. equi $\Delta m r x 1 \Delta m r x 2 \Delta m r x 3$ mutant (dark grey) to clofazimine (Clo) and norfloxacin (Nor). The concentration of each antibiotic used is shown in brackets $(\mu \mathrm{g} / \mathrm{ml})$. The diameter of the growth inhibition zones was measured to the nearest millimetre, and the mean \pm SD of three independent experiments was plotted. Two-way ANOVA and post-hoc Tukey's multiple comparison tests were performed to assess for statistical significance across comparisons of the wild type strain and the triple $m r x$-null mutant. $p$-value $<0.05\left(^{*}\right)$. (B) Ratiometric response of the Mrx1-roGFP2 biosensor. Fluorescence 405/490 ratio was calculated by confocal microscopy at different time points in presence of $10 \mu \mathrm{g} / \mathrm{mL}$ of norfloxacin. The results show the mean $\pm \mathrm{SD}$ of three independent experiments. 


\subsection{Combinatorial Antibiotherapy against R. equi with ROS-Generating Antimicrobials}

We considered our screening results as proof-of-principle of the feasibility of an antibiotherapy against $R$. equi based on antibiotics that generate oxidative stress. Consequently, we studied a possible synergistic effect of ROS-generating anti-R. equi compounds. We therefore analysed the combined effect of the most promising ROS-producing compounds against $R$. equi $103 S^{+}$in comparison to the commonly used combination of erythromycin and rifampicin (Figure 3). Interestingly, norfloxacin combined with either rifampicin, erythromycin, or vancomycin had an equivalent antimicrobial effect to the antibiotherapy based on erythromycin and rifampicin (Figure 3). This suggested that norfloxacin could be an important ROS-generating adjuvant to other anti-infectives against R. equi. In contrast, the oxidative stressing anti-tuberculosis drug clofazimine did not complement the antimicrobial effect of neither rifampicin nor erythromycin (Figure 3), confirming our screening results.

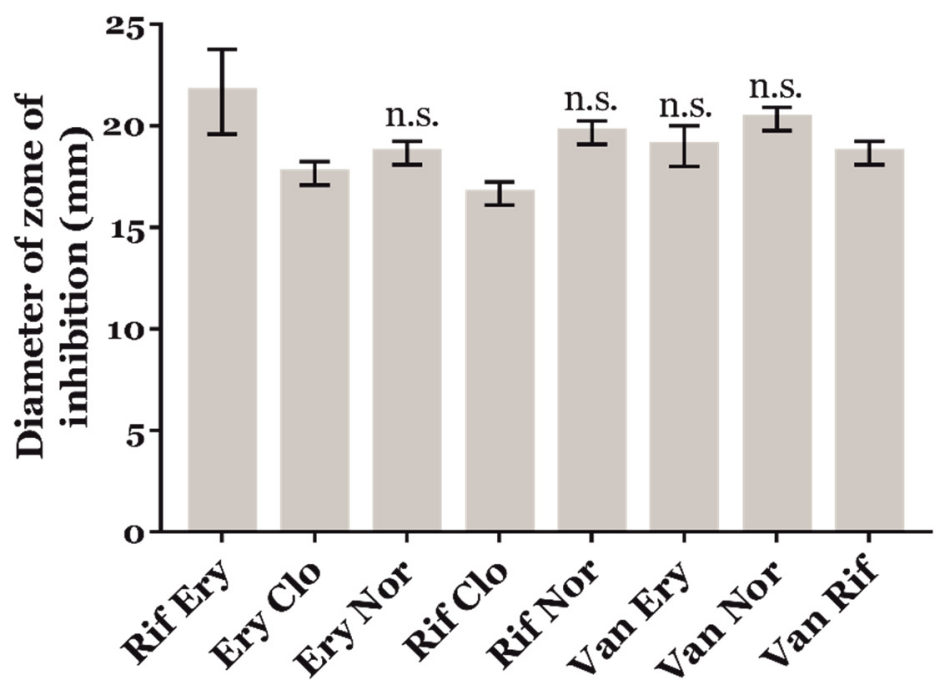

Figure 3. Antimicrobial susceptibility of R. equi $103 S^{+}$to $5 \mu \mathrm{g}$ of different combinations of antimicrobial compounds, including clofazimine (Clo), erythromycin (Ery), norfloxacin (Nor), rifampicin (Rif), and vancomycin (Van). The diameter of the growth inhibition zones was measured to the nearest millimetre, and the mean \pm SD of three independent experiments was plotted. Two-way ANOVA and post-hoc Tukey's multiple comparison tests were performed to assess for statistical significance across conditions. N.s.: Not significant.

In order to produce a clinically relevant analysis of the combined effect of norfloxacin with any of the other ROS-generating antimicrobials tested, we calculated the minimum inhibitory concentration (MIC) of different combinations of antibiotics following the guidelines of the Clinical \& Laboratory Standards Institute [10,21,29] (Table 1).

Table 1. MICs of different antibacterial compounds and their combinations against $R$. equi $103 S^{+}$. The data are resulting from three independent experiments with two technical replicates per assay.

\begin{tabular}{cc}
\hline Antibiotics & MIC \\
\hline Rifampicin & $0.4 \mu \mathrm{g} / \mathrm{mL}$ \\
Erythromycin & $1.3 \mu \mathrm{g} / \mathrm{mL}$ \\
Vancomycin & $1 \mu \mathrm{g} / \mathrm{mL}$ \\
Norfloxacin & $>10 \mu \mathrm{g} / \mathrm{mL}$ \\
Rifampicin + Erythromycin & $0.4+1.3 \mu \mathrm{g} / \mathrm{mL}$ \\
Rifampicin + Norfloxacin & $0.4+1 \mu \mathrm{g} / \mathrm{mL}$ \\
Erythromycin + Norfloxacin & $0.5+1 \mu \mathrm{g} / \mathrm{mL}$ \\
Rifampicin + Vancomycin & $0.05+0.25 \mu \mathrm{g} / \mathrm{mL}$ \\
Erythromycin + Vancomycin & $0.5+0.25 \mu \mathrm{g} / \mathrm{mL}$ \\
Vancomycin + Norfloxacin & $0.5+1 \mu \mathrm{g} / \mathrm{mL}$ \\
\hline
\end{tabular}


We first established the MIC of individual antibacterial compounds (Table 1). Surprisingly, norfloxacin alone did not generate a reportable MIC for $R$. equi $103 S^{+}$below $10 \mu \mathrm{g} / \mathrm{mL}$, despite being a broad-spectrum antimicrobial [30]. In addition, the combination of rifampicin and erythromycin did not generate a synergistic effect (Table 1$)$. However, low doses of norfloxacin $(1 \mu \mathrm{g} / \mathrm{mL}) \mathrm{combined}$ with subminimal inhibitory concentrations of erythromycin (2.6-fold lower than its individual MIC) or vancomycin (2-fold lower than its individual MIC) inhibited R. equi growth (Table 1), which suggested synergy. Moreover, a similar effect was detected with combinations of low doses of vancomycin and either rifampicin (8-fold lower than its individual MIC) or erythromycin (2.6-fold lower than its individual MIC; Table 1). Overall, our in vitro results suggested that norfloxacin and vancomycin might be very promising ROS-generating agents against $R$. equi. However, vancomycin is considered a last-resort drug and therefore its veterinary use is not recommended.

\subsection{Antimicrobial Activity of ROS-Generating Anti-Infectives against Intracellular R. equi}

Antibacterial compounds might have deleterious effects on mammalian cells [15]. Therefore, the use of ROS-generating antimicrobials against intracellular pathogens might lead to unexpected results during host-cell infection. Due to this, we studied the combinatorial antimicrobial activity of the ROS-generating drugs identified in our screening on the intracellular survival of R. equi (Figure 4). In these experiments, we initially used the minimal inhibitory concentrations determined before for each antibiotic or combinations of antibiotics (Table 1).

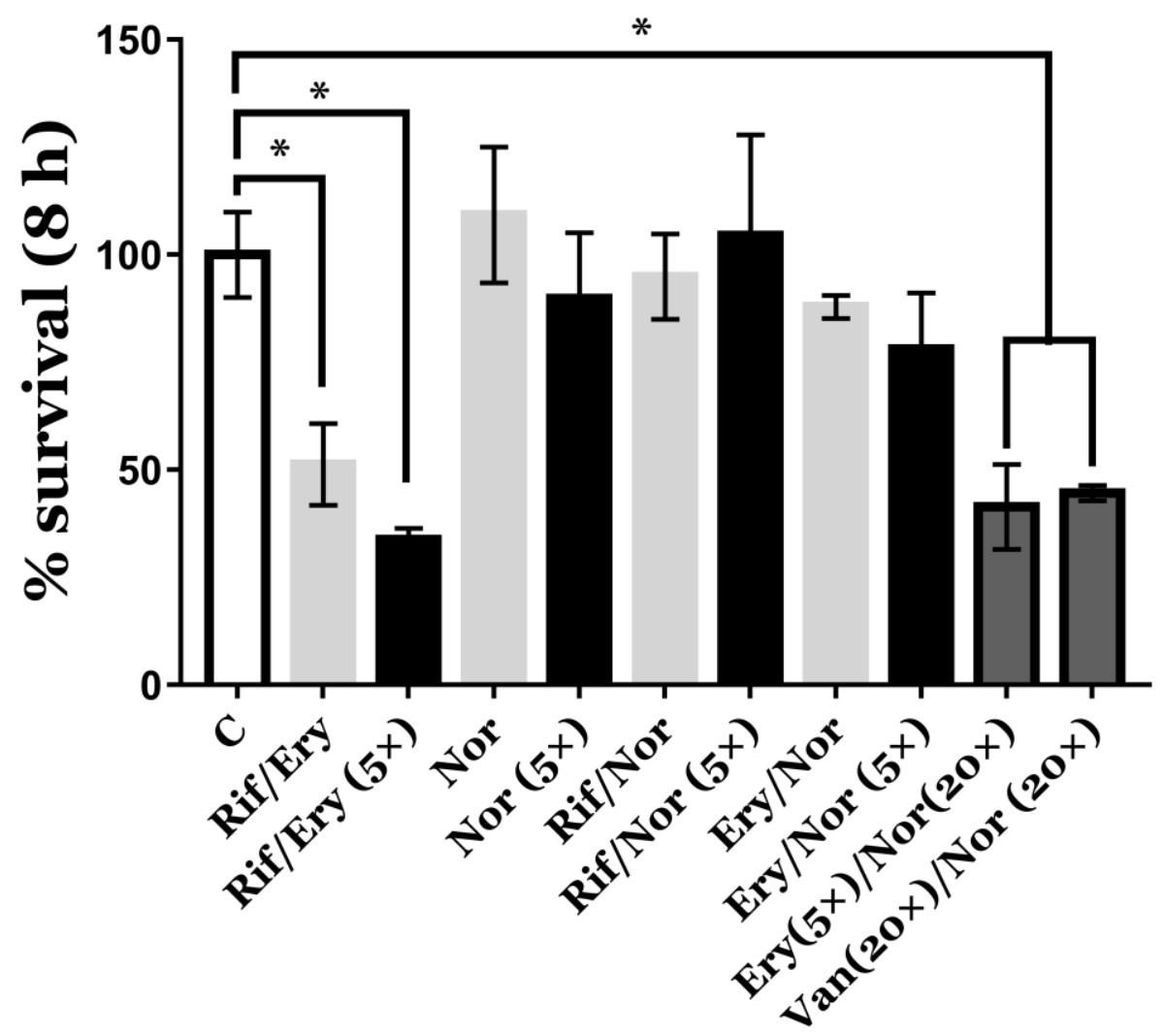

Figure 4. Antimicrobial effect of different combinatorial antibiotherapies against intracellular R. equi $103 S^{+}$infecting J774.A1 murine macrophages. Percentages of survival were quantified after $8 \mathrm{~h}$ of infection. Different combinations of rifampicin (Rif), erythromycin (Ery), norfloxacin (Nor), and vancomycin (Van) were compared against a negative control with gentamicin $(\mathrm{C})$. The concentration of some drug combinations was increased 5 -fold $(5 \times)$ or 20 -fold $(20 \times)$ their MIC. Two-way ANOVA and post-hoc Tukey's multiple comparison tests were performed to assess for statistical significance related to the number of CFU recovered from the negative control (C). $p$-value $<0.05\left(^{*}\right)$. 
However, the synergistic effect of norfloxacin in combination with other ROS-producing antimicrobials such as erythromycin or vancomycin was only reproduced against intracellular $R$. equi when the concentration of this antibiotic was raised 20-fold (Figure 4). Similar to vancomycin [31,32], the cellular uptake of norfloxacin in macrophages might be quite poor, which could be an important barrier for the use of this therapeutic strategy in vivo. However, the intracellular delivery of antibiotics could be facilitated by their liposomal encapsulation [31]. Moreover, the therapeutic index of the encapsulated antibiotics increases, while the drug toxicity is reduced due to their intracellular delivery. Consequently, lower plasma concentrations are required to achieve an effective antimicrobial activity against intracellular pathogens [31]. Accordingly, gentamicin encapsulated in liposomes efficiently eradicated R. equi in a mouse model of infection [32]. Unfortunately, liposomal gentamicin caused nephrotoxicity in foals [33], abolishing the potential veterinary use of this particular encapsulated antibiotic. Nevertheless, the use of liposomal antibiotics for the treatment of infections caused by intracellular pathogens is now widely recognized as an important and safe therapeutic strategy to increase the internalization of highly effective antibiotics with poor cellular uptake [34].

\section{Conclusions}

R. equi is an intracellular veterinary pathogen that is rapidly acquiring antimicrobial resistance to currently used anti-infective strategies [7,8]. Rifampicin resistance in $R$. equi is associated to mutations of the rpoB gene [10], while the lateral acquisition of erm (46) gene is driving the emergence of macrolide-resistant $R$. equi isolates in the United States [35].

With the aim to develop novel ROS-inspired antimicrobial therapies against this actinobacterial pathogen, here we developed a new screening strategy for the in vitro identification of antimicrobial compounds that might synergistically cause oxidative stress. Interestingly, norfloxacin might be a promising adjuvant to other ROS-generating antimicrobials currently used to treat $R$. equi infections. Norfloxacin is a drug with great repurposing potential, as it is commonly used to treat urinary infections in humans [30]. Therefore, these results might pave the way for the rapid development of novel antimicrobial strategies with important veterinary applications.

Author Contributions: L.M.M. and M.L. conceived the experiments; Á.M., J.A.G., L.M.M., and M.L. were involved in the design of the experiments and interpretation of the results; Á.M. performed the experiments, wrote the first draft of the manuscript, and analysed the data. All authors have read and agreed to the published version of the manuscript.

Funding: This research was funded by intramural funding from the University of Leon to L.M.M. and from the University of Roehampton to M.L. Á.M. was granted an "Ayuda Puente" from the University of Leon.

Acknowledgments: We thank Tobias Dick for permission to work with the roGFP2 biosensor.

Conflicts of Interest: The authors declare no conflict of interest.

\section{References}

1. Weinstock, D.M.; Brown, A.E. Rhodococcus equi: An emerging pathogen. Clin. Infect. Dis. 2002, 34, 1379-1385. [CrossRef] [PubMed]

2. Vázquez-Boland, J.A.; Giguère, S.; Hapeshi, A.; MacArthur, I.; Anastasi, E.; Valero-Rello, A. Rhodococcus equi: The many facets of a pathogenic actinomycete. Vet. Microbiol. 2013, 167, 9-33. [CrossRef] [PubMed]

3. Yamshchikov, A.V.; Schuetz, A.; Lyon, G.M. Rhodococcus equi infection. Lancet Infect. Dis. 2010, 10, 350-359. [CrossRef]

4. Kedlaya, I.; Ing, M.B.; Wong, S.S. Rhodococcus equi infections in immunocompetent hosts: Case report and review. Clin. Infect. Dis. 2001, 32, E39-E46. [CrossRef] [PubMed]

5. Muscatello, G.; Anderson, G.A.; Gilkerson, J.R.; Browning, G.F. Associations between the ecology of virulent Rhodococcus equi and the epidemiology of R. equi pneumonia on Australian thoroughbred farms. Appl. Environ. Microbiol. 2006, 72, 6152-6160. [CrossRef] [PubMed] 
6. Muscatello, G.; Gerbaud, S.; Kennedy, C.; Gilkerson, J.R.; Buckley, T.; Klay, M.; Leadon, D.P.; Browning, G.F. Comparison of concentrations of Rhodococcus equi and virulent $R$. equi in air of stables and paddocks on horse breeding farms in a temperate climate. Equine Vet. J. 2006, 38, 263-265. [CrossRef]

7. Huber, L.; Giguère, S.; Cohen, N.D.; Slovis, N.M.; Berghaus, L.; Greiter, M.; Hart, K.A. Identification of macrolide- and rifampicin-resistant Rhodococcus equi in environmental samples from equine breeding farms in central Kentucky during 2018. Vet. Microbiol. 2019, 232, 74-78. [CrossRef]

8. Huber, L.; Giguère, S.; Slovis, N.M.; Carter, C.N.; Barr, B.S.; Cohen, N.D.; Elam, J.; Erol, E.; Locke, S.J.; Phillips, E.D.; et al. Emergence of resistance to macrolides and rifampin in clinical isolates of Rhodococcus equi from foals in central Kentucky, 1995 to 2017. Antimicrob. Agents Chemother. 2019, 63, 1-6. [CrossRef]

9. Cisek, A.A.; Rzewuska, M.; Witkowski, L.; Binek, M. Antimicrobial resistance in Rhodococcus equi. Acta Biochim. Pol. 2014, 61, 633-638. [CrossRef]

10. Giguère, S.; Berghaus, L.J.; Willingham-Lane, J.N. Antimicrobial resistance in Rhodococcus equi. Microbiol. Spectrum. 2017, 5. [CrossRef]

11. Giles, C.; Vanniasinkam, T.; Ndi, S.; Barton, M.D. Rhodococcus equi (Prescottella equi) vaccines; the future of vaccine development. Equine Vet. J. 2015, 47, 510-518. [CrossRef] [PubMed]

12. Giguère, S.; Berghaus, L.J.; Lee, E.A. Activity of 10 antimicrobial agents against intracellular Rhodococcus equi. Vet. Microbiol. 2015, 178, 275-278. [CrossRef] [PubMed]

13. Higuchi, T.; Arakawa, T.; Hashikura, S.; Inui, T.; Senba, H.; Takai, S. Effect of prophylactic administration of hyperimmune plasma to prevent Rhodococcus equi infection on foals from endemically affected farms. J. Vet. Med. Ser. B 1999, 46, 641-648. [CrossRef]

14. Kohanski, M.A.; Dwyer, D.J.; Hayete, B.; Lawrence, C.A.; Collins, J.J. A common mechanism of cellular death induced by bactericidal antibiotics. Cell 2007, 130, 797-810. [CrossRef] [PubMed]

15. Kalghatgi, S.; Spina, C.S.; Costello, J.C.; Liesa, M.; Morones-Ramirez, J.R.; Slomovic, S.; Molina, A.; Shirihai, O.S.; Collins, J.J. Bactericidal antibiotics induce mitochondrial dysfunction and oxidative damage in mammalian cells. Sci. Transl. Med. 2013, 5, 192ra85. [CrossRef] [PubMed]

16. Kim, S.Y.; Park, C.; Jang, H.J.; Kim, B.O.; Bae, H.W.; Chung, I.Y.; Kim, E.S.; Cho, Y.H. Antibacterial strategies inspired by the oxidative stress and response networks. J. Microbiol. 2019, 57, 203-212. [CrossRef]

17. Mourenza, Á.; Bravo-Santano, N.; Pradal, I.; Gil, J.A.; Mateos, L.M.; Letek, M. Mycoredoxins are required for redox homeostasis and intracellular survival in the actinobacterial pathogen Rhodococcus equi. Antioxidants 2019, 8, 558. [CrossRef]

18. Tung, Q.N.; Loi, V.V.; Busche, T.; Nerlich, A.; Mieth, M.; Milse, J.; Kalinowski, J.; Hocke, A.C.; Antelmann, H. Stable integration of the Mrx1-roGFP2 biosensor to monitor dynamic changes of the mycothiol redox potential in Corynebacterium glutamicum. Redox Biol. 2019, 20, 514-525. [CrossRef]

19. Bhaskar, A.; Chawla, M.; Mehta, M.; Parikh, P.; Chandra, P.; Bhave, D.; Kumar, D.; Carroll, K.S.; Singh, A. Reengineering Redox sensitive GFP to measure mycothiol Redox potential of Mycobacterium tuberculosis during infection. PLoS Pathog. 2014, 10, e1003902. [CrossRef]

20. Gutscher, M.; Pauleau, A.-L.; Marty, L.; Brach, T.; Wabnitz, G.H.; Samstag, Y.; Meyer, A.J.; Dick, T.P. Real-time imaging of the intracellular glutathione redox potential. Nat. Methods 2008, 5, 553-559. [CrossRef]

21. Riesenberg, A.; Feßler, A.T.; Erol, E.; Prenger-Berninghoff, E.; Stamm, I.; Böse, R.; Heusinger, A.; Klarmann, D.; Werckenthin, C.; Schwarz, S. MICs of 32 antimicrobial agents for Rhodococcus equi isolates of animal origin. J. Antimicrob. Chemother. 2014, 69, 1045-1049. [CrossRef] [PubMed]

22. Letek, M.; González, P.; MacArthur, I.; Rodríguez, H.; Freeman, T.C.; Valero-Rello, A.; Blanco, M.; Buckley, T.; Cherevach, I.; Fahey, R.; et al. The genome of a pathogenic Rhodococcus: Cooptive virulence underpinned by key gene acquisitions. PLoS Genet. 2010, 6, e1001145. [CrossRef] [PubMed]

23. Van Loi, V.; Rossius, M.; Antelmann, H. Redox regulation by reversible protein S-thiolation in bacteria. Front. Microbiol. 2015, 6, 1-22. [CrossRef]

24. Bauer, A.W.; Kirby, W.M.M.; Sherris, J.C.; Turck, M. Antibiotic susceptibility testing by a standardized single disk method. Am. J. Clin. Pathol. 1966, 45, 493-496. [CrossRef]

25. Dwyer, D.J.; Kohanski, M.A.; Hayete, B.; Collins, J.J. Gyrase inhibitors induce an oxidative damage cellular death pathway in Escherichia coli. Mol. Syst. Biol. 2007, 3, 91. [CrossRef]

26. Yano, T.; Kassovska-Bratinova, S.; Shin Teh, J.; Winkler, J.; Sullivan, K.; Isaacs, A.; Schechter, N.M.; Rubin, H. Reduction of clofazimine by mycobacterial type $2 \mathrm{NADH}$ : Quinone Oxidoreductase: A pathway for the generation of bactericidal levels of reactive oxygen species. J. Biol. Chem. 2011, 286, 10276-10287. [CrossRef] 
27. Kottur, J.; Nair, D.T. Reactive oxygen species play an important role in the bactericidal activity of quinolone antibiotics. Angew. Chem. Int. Ed. 2016, 55, 2397-2400. [CrossRef]

28. Dwyer, D.J.; Belenky, P.A.; Yang, J.H.; MacDonald, C.I.; Martell, J.D.; Takahashi, N.; Chan, C.T.Y.; Lobritz, M.A.; Braff, D.; Schwarz, E.G.; et al. Antibiotics induce redox-related physiological alterations a part of their lethality. Proc. Natl. Acad. Sci. USA 2014, 111, E2100-E2109. [CrossRef]

29. Riesenberg, A.; Kaspar, H.; Feßler, A.T.; Werckenthin, C.; Schwarz, S. Susceptibility testing of Rhodococcus equi: An interlaboratory test. Vet. Microbiol. 2016, 194, 30-35. [CrossRef]

30. Chierentin, L.; Salgado, H.R.N. Review of properties and analytical methods for the determination of Norfloxacin. Crit. Rev. Anal. Chem. 2016, 46, 22-39. [CrossRef]

31. Sande, L.; Sanchez, M.; Montes, J.; Wolf, A.J.; Morgan, M.A.; Omri, A.; Liu, G.Y. Liposomal encapsulation of vancomycin improves killing of methicillin-resistant Staphylococcus aureus in a murine infection model. J. Antimicrob. Chemother. 2012, 67, 2191-2194. [CrossRef] [PubMed]

32. Burton, A.J.; Giguère, S.; Berghaus, L.J.; Hondalus, M.K.; Arnold, R.D. Efficacy of liposomal gentamicin against Rhodococcus equi in a mouse infection model and colocalization with $R$. equi in equine alveolar macrophages. Vet. Microbiol. 2015, 176, 292-300. [CrossRef] [PubMed]

33. Cohen, N.D.; Giguère, S.; Burton, A.J.; Rocha, J.N.; Berghaus, L.J.; Brake, C.N.; Bordin, A.I.; Coleman, M.C. Use of liposomal gentamicin for treatment of 5 foals with experimentally induced Rhodococcus equi pneumonia. J. Vet. Intern. Med. 2016, 30, 322-325. [CrossRef]

34. Abed, N.; Couvreur, P. Nanocarriers for antibiotics: A promising solution to treat intracellular bacterial infections. Int. J. Antimicrob. Agents 2014, 43, 485-496. [CrossRef]

35. Álvarez-Narváez, S.; Giguère, S.; Anastasi, E.; Hearn, J.; Scortti, M.; Vázquez-Boland, J.A. Clonal confinement of a highly mobile resistance element driven by combination therapy in Rhodococcus equi. mBio 2019, 10, e02260-19. [CrossRef] [PubMed]

(C) 2020 by the authors. Licensee MDPI, Basel, Switzerland. This article is an open access article distributed under the terms and conditions of the Creative Commons Attribution (CC BY) license (http://creativecommons.org/licenses/by/4.0/). 